\title{
Efficient Solutions of Multidimensional Sixth-Order Boundary Value Problems Using Symmetric Generalized Jacobi-Galerkin Method
}

\author{
E. H. Doha and W. M. Abd-Elhameed \\ Department of Mathematics, Faculty of Science, Cairo University, Giza 12613, Egypt \\ Correspondence should be addressed to W. M. Abd-Elhameed, walee_9@yahoo.com
}

Received 16 December 2011; Accepted 20 March 2012

Academic Editor: Svatoslav Staněk

Copyright (C) 2012 E. H. Doha and W. M. Abd-Elhameed. This is an open access article distributed under the Creative Commons Attribution License, which permits unrestricted use, distribution, and reproduction in any medium, provided the original work is properly cited.

This paper presents some efficient spectral algorithms for solving linear sixth-order two-point boundary value problems in one dimension based on the application of the Galerkin method. The proposed algorithms are extended to solve the two-dimensional sixth-order differential equations. A family of symmetric generalized Jacobi polynomials is introduced and used as basic functions. The algorithms lead to linear systems with specially structured matrices that can be efficiently inverted. The various matrix systems resulting from the proposed algorithms are carefully investigated, especially their condition numbers and their complexities. These algorithms are extensions to some of the algorithms proposed by Doha and Abd-Elhameed (2002) and Doha and Bhrawy (2008) for second- and fourth-order elliptic equations, respectively. Three numerical results are presented to demonstrate the efficiency and the applicability of the proposed algorithms.

\section{Introduction}

The classical Jacobi polynomials $P_{n}^{(\alpha, \beta)}(x)$ play important roles in mathematical analysis and its applications (see, e.g., [1-4]). In particular, the Legendre, the Chebyshev, and the ultraspherical polynomials have played important roles in spectral methods for partial differential equations (see, e.g., $[5,6])$. It is proven that the Jacobi polynomials are precisely the only polynomials arising as eigenfunctions of a singular Sturm-Liouville problem, (see [7, Section 9.2]). This class of polynomials comprises all the polynomial solution to singular Sturm-Liouville problems on $[-1,1]$.

Spectral methods have developed rapidly over the past four decades. Their fascinating merit is the high accuracy; they have been applied successfully to numerical simulations of 
many problems in science and engineering. The spectral methods that are mostly used are based on the Chebyshev and Legendre approximations.

Sixth-order boundary value problems arise in astrophysics, that is, the narrow convecting layers bounded by stable layers which are believed to surround $A$-type stars may be modelled by sixth-order boundary value problems (see [8]).

Chandrasekhar [9] determined that when an infinite horizontal layer of fluid is heated from below and is under the action of rotation, instability sets in. When this instability is as ordinary convection, the ordinary differential equation is sixth-order. Agarwal [10] presented the theorems stating the conditions for the existence and uniqueness of solutions of sixth order boundary value problems, while no numerical methods are contained therein. In [11] Bhrawy discussed the solution of sixth-order boundary value problems using Legendre Galerkin method. Lamnii et al. [12] used Spline collocation method for solving linear sixthorder boundary-value problems. Boutayeb and Twizell [8] developed a family of numerical methods for the solution of special nonlinear sixth-order boundary value problems. Siddiqi and Twizell [13] presented the solution of sixth order boundary value problem using the sextic spline. El-Gamel et al. [14] used Sinc-Galerkin method for the solutions of sixth order boundary value problems.

Guo et al. [15] extended the definition of the classical Jacobi polynomials with indexes

$\alpha, \beta>-1$ to allow $\alpha$ and /or $\beta$ to be negative integers. They showed also that the generalized Jacobi polynomials, with indexes corresponding to the number of boundary conditions in a given partial differential equation, are the natural basis functions for the spectral approximation of this equation. Moreover, it is shown that the use of generalized Jacobi polynomials not only simplified the numerical analysis for the spectral approximations of differential equations, but also led to very efficient numerical algorithms.

From the numerical point of view, Doha and Abd-Elhameed [16, 17], Doha and Bhrawy [18], and Doha et al. [19, 20] have constructed efficient spectral Galerkin algorithms using compact combinations of orthogonal polynomials for solving elliptic equations of second-, third-, fourth-, and fifth-order equations in various situations.

In this paper we are concerned with the direct solution techniques for sixthorder two-point boundary value problems, using symmetric generalized Jacobi-Galerkin approximations. Our algorithms lead to discrete linear systems with specially structured matrices that can be efficiently inverted.

We organize the materials of this paper as follows. In Section 2, we give some properties of classical and generalized Jacobi polynomials. In Section 3, we discuss two algorithms for solving the sixth-order elliptic linear differential equations subject to homogeneous and nonhomogeneous boundary conditions using symmetric generalized Jacobi Galerkin method (SGJGM). In Section 4, we explain how the idea of Section 3 can be extended to handle the sixth-order two dimensional differential equations. Three Numerical examples are given in Section 5 to show the efficiency of our algorithms. Some Concluding remarks are given in Section 6.

\section{Some Properties of Classical and Generalized Jacobi Polynomials}

\subsection{Classical Jacobi Polynomials}

The classical Jacobi polynomials, associated with the real parameters $(\alpha>-1, \beta>-1)$ (see $[4,21])$, are a sequence of polynomials, $P_{n}^{(\alpha, \beta)}(x), x \in(-1,1)(n=0,1,2, \ldots)$, each, 
respectively, of degree $n$. For our present purposes, it is more convenient to introduce the normalized orthogonal polynomials $R_{n}^{(\alpha, \beta)}(x)=\left(P_{n}^{(\alpha, \beta)}(x)\right) /\left(P_{n}^{(\alpha, \beta)}(1)\right)$. This means that $R_{n}^{(\alpha, \beta)}(x)=(n ! \Gamma(\alpha+1)) /(\Gamma(n+\alpha+1)) P_{n}^{(\alpha, \beta)}(x)$. In such case $R_{n}^{(\alpha-(1 / 2), \alpha-(1 / 2))}(x)$ is identical to the ultraspherical polynomials $C_{n}^{(\alpha)}(x)$, and the polynomials $R_{n}^{(\alpha, \beta)}(x)$ may be generated using the recurrence relation

$$
\begin{aligned}
& 2(n+\lambda)(n+\alpha+1)(2 n+\lambda-1) R_{n+1}^{(\alpha, \beta)}(x)=(2 n+\lambda-1)_{3} x R_{n}^{(\alpha, \beta)}(x) \\
& \quad+\left(\alpha^{2}-\beta^{2}\right)(2 n+\lambda) R_{n}^{(\alpha, \beta)}(x)-2 n(n+\beta)(2 n+\lambda+1) R_{n-1}^{(\alpha, \beta)}(x), \quad n=1,2, \ldots,
\end{aligned}
$$

starting from $R_{0}^{(\alpha, \beta)}(x)=1$ and $R_{1}^{(\alpha, \beta)}(x)=(1 / 2(\alpha+1))[\alpha-\beta+(\lambda+1) x]$, or obtained from Rodrigues' formula

$$
R_{n}^{(\alpha, \beta)}(x)=\left(\frac{-1}{2}\right)^{n} \frac{\Gamma(\alpha+1)}{\Gamma(n+\alpha+1)}(1-x)^{-\alpha}(1+x)^{-\beta} D^{n}\left[(1-x)^{\alpha+n}(1+x)^{\beta+n}\right]
$$

where

$$
\lambda=\alpha+\beta+1, \quad(a)_{k}=\frac{\Gamma(a+k)}{\Gamma(a)}, \quad D=\frac{d}{d x}
$$

and satisfy the orthogonality relation

$$
\int_{-1}^{1}(1-x)^{\alpha}(1+x)^{\beta} R_{m}^{(\alpha, \beta)}(x) R_{n}^{(\alpha, \beta)}(x) d x= \begin{cases}0, & m \neq n, \\ h_{n}^{\alpha, \beta}, & m=n,\end{cases}
$$

where

$$
h_{n}^{\alpha, \beta}=\frac{2^{\lambda} n ! \Gamma(n+\beta+1)[\Gamma(\alpha+1)]^{2}}{(2 n+\lambda) \Gamma(n+\lambda) \Gamma(n+\alpha+1)} .
$$

These polynomials are eigenfunctions of the following singular Sturm-Liouville equation:

$$
\left(1-x^{2}\right) \phi^{\prime \prime}(x)+[\beta-\alpha-(\lambda+1) x] \phi^{\prime}(x)+n(n+\lambda) \phi(x)=0 .
$$

The following relations will be of important use later:

$$
\begin{gathered}
\left(1-x^{2}\right) R_{k-1}^{(\alpha+1, \alpha+1)}(x)=\frac{2(\alpha+1)}{2 k+2 \alpha+1}\left[R_{k-1}^{(\alpha, \alpha)}(x)-R_{k+1}^{(\alpha, \alpha)}(x)\right] \\
D R_{k}^{(\alpha, \alpha)}(x)=\frac{k(k+2 \alpha+1)}{2(\alpha+1)} R_{k-1}^{(\alpha+1, \alpha+1)}(x), \quad k=1,2, \ldots
\end{gathered}
$$

The following two theorems are needed hereafter. 
Theorem 2.1. The qth derivative of the symmetric normalized Jacobi polynomial $R_{n}^{(\alpha, \alpha)}(x)$ is given explicitly by

$$
\begin{aligned}
D^{q} & R_{k}^{(\alpha, \alpha)}(x) \\
= & \frac{2^{q} k !}{(q-1) ! \Gamma(k+2 \alpha+1)} \\
& \times \sum_{\substack{m=0 \\
(k+m-q) \text { even }}}^{k-q} \frac{(m+\alpha+1 / 2) \Gamma(m+2 \alpha+1)((k-m+q-2) / 2) ! \Gamma((k+m+q+2 \alpha+1) / 2)}{m !((k-q-m) / 2) ! \Gamma((k+m-q+2 \alpha+3) / 2)} \\
& \times R_{m}^{(\alpha, \alpha)}(x), \quad k \geq q .
\end{aligned}
$$

(For the proof of Theorem 2.1, see [22].)

Theorem 2.2. If one defines the $q$ times repeated integration of the symmetric normalized Jacobi polynomials $R_{k}^{(\alpha, \alpha)}(x)$ by

$$
I_{k}^{(q, \alpha)}(x)=\overbrace{\iint \ldots \int}^{q \text { times }} R_{k}^{(\alpha, \alpha)}(x) \overbrace{d x d x \cdots d x}^{q \text { times }}
$$

then

$$
\begin{aligned}
I_{k}^{(q, \alpha)}(x)= & \frac{2^{-q} k !}{\Gamma(k+2 \alpha+1)} \sum_{j=0}^{q} \frac{(-1)^{j}\left(\begin{array}{c}
q \\
j
\end{array}\right) \Gamma(k-j+\alpha+1 / 2) \Gamma(k+q-2 j+2 \alpha+1)}{(k+q-2 j) ! \Gamma(k+q-j+\alpha+3 / 2)} \\
& \times\left(k+q-2 j+\alpha+\frac{1}{2}\right) R_{k+q-2 j}^{(\alpha, \alpha)}(x), \quad q \geq 0, k \geq q+1 \text { for } \alpha=0, q \geq 0, k \geq q \text { for } \alpha \neq 0 .
\end{aligned}
$$

(For the proof of Theorem 2.2, see [23].)

Also, the following two lemmas are needed in the sequel.

Lemma 2.3. For all $k \geq 0$, one has

$$
-D^{2}\left[\left(1-x^{2}\right) R_{k}^{(1,1)}(x)\right]=(k+1)(k+2) R_{k}^{(1,1)}(x) .
$$

(For the proof of Lemma 2.3, see [16].)

Lemma 2.4. For all $k \geq 0$, one has

$$
D^{4}\left[\left(1-x^{2}\right)^{2} R_{k}^{(2,2)}(x)\right]=(k+1)_{4} R_{k}^{(2,2)}(x) .
$$


Proof. Setting $\alpha=\beta=2$ in relation (2.7), we get

$$
\left(1-x^{2}\right) R_{k}^{(2,2)}(x)=\frac{4}{2 k+5}\left[R_{k}^{(1,1)}(x)-R_{k+2}^{(1,1)}(x)\right] .
$$

Making use of this relation and with the aid of Lemma 2.3, we obtain

$$
D^{4}\left[\left(1-x^{2}\right)^{2} R_{k}^{(2,2)}(x)\right]=\frac{4}{2 k+5} D^{2}\left[(k+3)(k+4) R_{k+2}^{(1,1)}(x)-(k+1)(k+2) R_{k}^{(1,1)}(x)\right],
$$

which in turn gives with the aid of relation (2.8)

$$
D^{4}\left[\left(1-x^{2}\right)^{2} R_{k}^{(2,2)}(x)\right]=\frac{1}{2 k+5} D\left[(k+2)_{4} R_{k+1}^{(2,2)}(x)-(k)_{4} R_{k-1}^{(2,2)}(x)\right] .
$$

Finally, from relation (2.9) (for $q=1$ and $\alpha=2$ ), we get

$$
D^{4}\left[\left(1-x^{2}\right)^{2} R_{k}^{(2,2)}(x)\right]=(k+1)_{4} R_{k}^{(2,2)}(x) .
$$

This completes the proof of Lemma 2.4.

\subsection{Generalized Jacobi Polynomials}

Following Guo et al. [15], we define a family of generalized Jacobi polynomials/functions with indexes $\alpha, \beta \in \mathbb{R}$.

Let $w^{\alpha, \beta}(x)=(1-x)^{\alpha}(1+x)^{\beta}$. We denote by $L_{w^{\alpha, \beta}}^{2}(-1,1)$ the weighted $L^{2}$ space with inner product:

$$
(u, v)_{w^{\alpha, \beta}}(x):=\int_{I} u(x) v(x) w^{\alpha, \beta}(x) d x
$$

and the associated norm $\|u\|_{w^{\alpha, \beta}}=(u, u)_{w^{\alpha, \beta}}^{1 / 2}$. We are interested in defining Jacobi polynomials with indexes $\alpha$ and/or $\beta \leq-1$, referred hereafter as generalized Jacobi polynomials (GJPs), in such a way that they satisfy some selected properties that are essentially relevant to spectral approximations. In this work, we will restrict our attention to the cases when $\alpha$ and $\beta$ are negative integers.

Let $\ell, m \in \mathbb{Z}$ (the set of all integers), and define

$$
J_{k}^{(\ell, m)}(x)= \begin{cases}(1-x)^{-\ell}(1+x)^{-m} R_{k-k_{0}}^{(-\ell,-m)}(x), & k_{0}=-(\ell+m), \ell, m \leq-1, \\ (1-x)^{-\ell} R_{k-k_{0}}^{(-\ell)}(x), & k_{0}=-\ell, \ell \leq-1, m>-1, \\ (1+x)^{-m} R_{k-k_{0}}^{(\ell,-m)}(x), & k_{0}=-m, \ell>-1, m \leq-1, \\ R_{k-k_{0}}^{(\ell, m)}(x), & k_{0}=0, \ell, m>-1 .\end{cases}
$$


An important property of the GJPs is that for $\ell, m \in \mathbb{Z}^{+}$,

$$
\begin{aligned}
& D^{i} J_{k}^{(-\ell,-m)}(1)=0, \quad i=0,1, \ldots, \ell-1, \\
& D^{j} J_{k}^{(-\ell,-m)}(-1)=0, \quad j=0,1, \ldots, m-1 .
\end{aligned}
$$

Using relation (2.7), and after performing some manipulation, $J_{k}^{(-3,-3)}(x)$ can be written in terms of Legendre polynomials as:

$$
\begin{aligned}
J_{k}^{(-3,-3)}(x)= & \frac{48}{(2 k-9)(2 k-7)(2 k-5)} \\
& +\left[L_{k-6}(x)-\frac{3(2 k-7)}{2 k-3} L_{k-4}(x)+\frac{3(2 k-9)}{2 k-1} L_{k-2}(x)-\frac{(2 k-7)(2 k-9)}{(2 k-3)(2 k-1)} L_{k}(x)\right]
\end{aligned}
$$

\section{Spectral-Galerkin Algorithms for One-Dimensional Sixth-Order Equations}

In this section, we are interested in using SGJGM to solve the sixth-order two-point boundary value problems in one dimension subject to homogeneous and nonhomogeneous boundary conditions.

\subsection{Homogeneous Boundary Conditions}

Let us consider the sixth-order differential equation

$$
-u^{(6)}(x)+\sum_{q=1}^{6} \delta_{q} \eta_{6-q} u^{(6-q)}(x)=f(x), \quad x \in(-1,1),
$$

subject to the homogeneous boundary conditions

$$
u^{(j)}( \pm 1)=0, \quad j=0,1,2
$$

where $u^{(j)}(x)$ denotes the $j$ th derivative of $u(x)$ with respect to $x$ and $\left\{\eta_{6-q}, q=1, \ldots, 6\right\}$ are positive constants, and

$$
\delta_{q}= \begin{cases}(-1)^{1+q / 2}, & q \text { even } \\ 1, & q \text { odd }\end{cases}
$$

Let us denote $H_{w}^{r}(I)(r=0,1,2, \ldots)$, as the weighted Sobolev spaces, whose inner products and norms are denoted by $(\cdot, \cdot)_{r, w}$ and $\|\cdot\|_{r, w}$, respectively. To account for homogeneous 
boundary conditions, we define

$$
H_{0, w}^{3}(I)=\left\{v \in H_{w}^{3}(I): v^{(j)}( \pm 1)=0,0 \leq j \leq 2\right\},
$$

where $v^{(j)}(x)=\left(d^{j} v\right) /\left(d x^{j}\right)$. The superscript $w$ will be omitted in case of $w=1$.

Let $P_{N}$ be the space of all polynomials of degree less than or equal to $N$. Setting $V_{N}=$ $P_{N} \cap H_{0}^{3}(I)$, then

$$
V_{N}:=\operatorname{span}\left\{J_{6}^{(-3,-3)}(x), J_{7}^{(-3,-3)}(x), \ldots, J_{N}^{(-3,-3)}(x)\right\} .
$$

The symmetric generalized Jacobi-Galerkin procedure for solving (3.1)-(3.2) is to find $u_{N} \in$ $V_{N}$ such that

$$
\left(-D^{6} u_{N}(x), v(x)\right)+\sum_{q=1}^{6} \delta_{q} \eta_{6-q}\left(D^{6-q} u_{N}(x), v(x)\right)=(f(x), v(x)), \quad \forall v \in V_{N}
$$

where $(u, v)=\int_{-1}^{1} u v d x$ is the scalar inner product in the space $L^{2}(-1,1)$.

\subsection{The Choice of Basis Functions}

We choose the basis functions of expansion to be

$$
\phi_{k}(x)=J_{k+6}^{(-3,-3)}(x)=\left(1-x^{2}\right)^{3} R_{k}^{(3,3)}(x), \quad k=0,1, \ldots, N-6,
$$

which fulfills the boundary conditions (3.2).

It is obvious that $\left\{\phi_{k}(x)\right\}$ are linearly independent. Therefore, we have

$$
V_{N}=\operatorname{span}\left\{\phi_{k}(x): k=0,1,2, \ldots, N-6\right\}
$$

Now, the following two lemmas are needed hereafter.

Lemma 3.1. For all $k \geq 0$, one has

$$
-D^{6}\left[J_{k+6}^{(-3,-3)}(x)\right]=(k+1)_{6} R_{k}^{(3,3)}(x) .
$$

Proof. Setting $\alpha=\beta=3$ in relation to (2.7), we get

$$
\left(1-x^{2}\right) R_{k}^{(3,3)}(x)=\frac{6}{2 k+7}\left[R_{k}^{(2,2)}(x)-R_{k+2}^{(2,2)}(x)\right] .
$$


Making use of this relation and with the aid of Lemma 2.4, we obtain

$$
-D^{6}\left[J_{k+6}^{(-3,-3)}(x)\right]=\frac{6}{2 k+7} D^{2}\left[(k+3)_{4} R_{k+2}^{(2,2)}(x)-(k+1)_{4} R_{k}^{(2,2)}(x)\right]
$$

The last relation with the aid of the two relations (2.8) and (2.9) yields

$$
-D^{6}\left[J_{k+6}^{(-3,-3)}(x)\right]=(k+1)_{6} R_{k}^{(3,3)}(x) .
$$

This completes the proof of Lemma 3.1.

Lemma 3.2. For all $k \geq 0$, one has

$$
D^{6-q}\left[J_{k+6}^{(-3,-3)}(x)\right]=\sum_{j=0}^{q} d_{j, k, q} R_{k-2 j+q^{\prime}}^{(3,3)} \quad 1 \leq q \leq 6,
$$

where

$$
d_{j, k, q}=\frac{(-1)^{j+1}\left(\begin{array}{c}
q \\
j
\end{array}\right) \Gamma(k-j+(7 / 2))(k+q-2 j+6) !(k+q-2 j+(7 / 2))}{2^{q}(k+q-2 j) ! \Gamma(k+q-j+(9 / 2))} .
$$

Proof. Integrating formula (3.9) $q$ times, $q \in\{1,2, \ldots, 6\}$, and with the aid of relation (2.11) (in case of $\alpha=3$ ), we obtain the desired formula.

Based on the results of the two Lemmas 3.1 and 3.2, we are able to state and prove the following two theorems.

Theorem 3.3. One has, for arbitrary constants $a_{k}$,

$$
-D^{6}\left[\sum_{k=0}^{N-6} a_{k} J_{k+6}^{(-3,-3)}(x)\right]=\sum_{k=0}^{N-6} b_{k} R_{k}^{(3,3)}(x)
$$

where

$$
b_{k}=(k+1)_{6} a_{k} .
$$

Theorem 3.4. One has, for arbitrary constants $a_{k}$, and $1 \leq q \leq 6$,

$$
D^{6-q}\left[\sum_{k=0}^{N-6} a_{k} J_{k+6}^{(-3,-3)}(x)\right]=\sum_{k=0}^{N+q-6} r_{k, q} R_{k}^{(3,3)}(x),
$$


where

$$
r_{k, q}=\sum_{i=0}^{q} d_{q-i, k+q-2 i, q} a_{k+q-2 i}
$$

Now, the application of Galerkin method to (3.1) gives

$$
\left(-D^{6} u_{N}(x)+\sum_{q=1}^{6} \delta_{q} \eta_{6-q} D^{6-q} u_{N}(x), \phi_{k}(x)\right)=\left(f(x), \phi_{k}(x)\right)
$$

where

$$
u_{N}(x)=\sum_{k=0}^{N-6} a_{k} \phi_{k}(x), \quad \phi_{k}(x)=J_{k+6}^{(-3,-3)}(x), \quad k=0,1, \ldots, N-6 .
$$

The variational formulation (3.19) is equivalent to

$$
\begin{gathered}
\left(-D^{6} u_{N}(x), R_{k}^{(3,3)}(x)\right)_{w^{3,3}(x)}+\sum_{q=1}^{6} \delta_{q} \eta_{6-q}\left(D^{6-q} u_{N}(x), R_{k}^{(3,3)}(x)\right)_{w^{3,3}(x)} \\
=\left(f(x), R_{k}^{(3,3)}(x)\right)_{w^{3,3}(x)^{\prime}} \quad w^{3,3}(x)=\left(1-x^{2}\right)^{3} .
\end{gathered}
$$

Substitution of formulae (3.15) and (3.17) into (3.21) yields

$$
\left(\sum_{j=0}^{N-6} b_{j} R_{j}^{(3,3)}(x)+\sum_{q=1}^{6} \sum_{j=0}^{N+q-6} \delta_{q} \eta_{6-q} r_{j, q} R_{j}^{(3,3)}(x), R_{k}^{(3,3)}(x)\right)_{w^{3,3}(x)}=\left(f(x), R_{k}^{(3,3)}(x)\right)_{w^{3,3}(x)^{\prime}}
$$

where $b_{k}$ and $r_{k, q}$ are as given by (3.16) and (3.18), respectively.

Now, if we apply the orthogonality relation of $R_{k}^{(3,3)}(x)$ on (3.22), we obtain the following linear system of equations:

$$
\left(b_{k}+\sum_{q=1}^{6} \delta_{q} \eta_{6-q} r_{k, q}\right) h_{k}^{3,3}=f_{k} ; \quad k=0,1, \ldots, N-6,
$$

where

$$
\begin{gathered}
f_{k}=\left(f(x), R_{k}^{(3,3)}(x)\right)_{w^{3,3}(x)^{\prime}} \\
h_{k}^{3,3}=\frac{4608}{(k+1)_{6}(2 k+7)} .
\end{gathered}
$$


The linear system (3.23) may be put in the form

$$
\left(b_{k}+\sum_{q=1}^{6} \delta_{q} \eta_{6-q} r_{k, q}\right)=f_{k^{\prime}}^{*} \quad k=0,1, \ldots, N-6,
$$

where

$$
f_{k}^{*}=\frac{f_{k}}{h_{k}^{3,3}}
$$

This system of equations may be put in the matrix form

$$
\left(B+\sum_{q=1}^{6} \eta_{6-q} G_{6-q}\right) \mathbf{a}=\mathbf{f}^{*},
$$

where

$$
\mathbf{a}=\left(a_{0}, a_{1}, \ldots, a_{N-6}\right)^{T}, \quad \mathbf{f}^{*}=\left(f_{0}^{*}, f_{1}^{*}, \ldots, f_{N-6}^{*}\right)^{T},
$$

and the nonzero elements of the matrices $B$ and $G_{6-q}, 1 \leq q \leq 6$ are given explicitly in the following theorem.

Theorem 3.5. If $u_{N}(x)=\sum_{k=0}^{N-6} a_{k} J_{k+6}^{(-3,-3)}(x)$ is the symmetric generalized Jacobi-Galerkin approximation to (3.1)-(3.2), then the expansion coefficients $\left\{a_{k}: k=0,1, \ldots, N-6\right\}$ satisfy the matrix system (3.28), where the nonzero elements of the matrices $B=\left(b_{k j}\right)$ and $G_{6-q}=\left(g_{k j}^{6-q}\right)=$ $\left(\delta_{q} r_{k j}^{6-q}\right), 0 \leq k, j \leq N-6,1 \leq q \leq 6$ are given as:

$$
\begin{gathered}
b_{k k}=(k+1)_{6} \\
g_{k, k+q-2 i}^{6-q}=\frac{\delta_{q}(-1)^{q+i+1}(2 k+7)(k+6) ! q ! \Gamma(-i+k+(7 / 2))}{2^{q+1} i ! k !(q-i) ! \Gamma(k-i+q+(9 / 2))}, \quad 0 \leq i \leq q .
\end{gathered}
$$

It is worthy to note here that the case corresponding to $\eta_{6-q}=0,1 \leq q \leq 6$ leads to a linear system with diagonal matrix. The result for such case is summarized in the following important corollary.

Corollary 3.6. If $u_{N}(x)=\sum_{k=0}^{N-6} a_{k} J_{k+6}^{(-3,-3)}(x)$ and $\eta_{6-q}=0,1 \leq q \leq 6$, is the symmetric generalized Jacobi-Galerkin approximation to problem (3.1)-(3.2), then the expansion coefficients $\left\{a_{k}: k=0,1, \ldots, N-6\right\}$ are given explicitly by

$$
a_{k}=\frac{2 k+7}{4608} \int_{-1}^{1}\left(1-x^{2}\right)^{3} f(x) R_{k}^{(3,3)}(x), \quad k=0,1, \ldots, N-6
$$


Table 1: Condition numbers for the two matrices $B$ and $D$.

\begin{tabular}{lcccc}
\hline$N$ & Cond $(B)$ & Cond $(D)$ & Cond $(B) / N^{6}$ & Cond $(D) / N^{6}$ \\
\hline 16 & 8008 & 7627.89 & $4.773 \cdot 10^{-4}$ & $4.547 \cdot 10^{-4}$ \\
32 & 906192 & 860739 & $8.439 \cdot 10^{-4}$ & $8.016 \cdot 10^{-4}$ \\
64 & $7.497 \cdot 10^{7}$ & $7.117 \cdot 10^{7}$ & $1.091 \cdot 10^{-3}$ & $1.035 \cdot 10^{-3}$ \\
128 & $5.424 \cdot 10^{9}$ & $5.148 \cdot 10^{9}$ & $1.233 \cdot 10^{-3}$ & $1.171 \cdot 10^{-3}$ \\
256 & $3.685 \cdot 10^{11}$ & $3.497 \cdot 10^{11}$ & $1.309 \cdot 10^{-3}$ & $1.243 \cdot 10^{-3}$ \\
\hline
\end{tabular}

Remark 3.7. Each of the matrices $G_{6-q}, q=1,2, \ldots, 6$ in (3.28) is a band matrix whose total number of nonzero diagonals upper or lower the main diagonal does not exceed $q$. Thus the coefficient matrix $D=B+\sum_{q=1}^{6} \eta_{6-q} G_{6-q}$ is seven-band matrix at most. This special structure of $D$ simplifies greatly the solution of the linear system (3.28). The system in such case can be factorized by $L U$-decomposition and the number of operations necessary to construct this factorization is of order $78(N-5)$, and the number of operations needed to solve the two triangular systems is of order $25(N-5)$.

Note

The total number of operations mentioned in the previous discussion includes the number of all subtractions, additions, divisions, and multiplications (see [24]).

\subsection{Condition Number}

For the direct collocation method, the condition number behaves like $O\left(N^{12}\right)(N$ : maximal degree of polynomials). In this paper we obtain an improved condition number with $O\left(N^{6}\right)$. The advantages with respect to propagation of rounding errors are demonstrated.

For GJGM, the resulting system from the equation $-u^{(6)}=f(x)$ is $B \mathbf{a}=\mathbf{f}^{*}$, where the matrix $B$ is a diagonal matrix whose elements are $b_{k k}=(k+1)_{6}$. Thus we note that the condition number of the matrix $B$ behaves like $O\left(k^{6}\right)$ for large values of $k$. Moreover, if we add $\sum_{q=1}^{6} \eta_{6-q} G_{6-q}$ to the matrix $B$, then we find that the eigenvalues of matrix $D=$ $B+\sum_{q=1}^{6} \eta_{6-q} G_{6-q}\left(\eta_{6-q}=1,(1 \leq q \leq 6)\right)$ are all real positive and the effect of these additions does not significantly change the values of the condition number for the system. This means that matrix $B$, which resulted from the highest derivatives of the differential equations under investigation, play the most important role in the propagation of the roundoff errors. In Table 1 we list the values of the condition numbers for the two matrices $B$ and $D$.

\subsection{Nonhomogeneous Boundary Conditions}

Let us consider the sixth-order differential equation,

$$
-u^{(6)}(x)+\sum_{q=1}^{6} \delta_{q} \eta_{6-q} u^{(6-q)}(x)=f(x), \quad x \in(-1,1)
$$


subject to the nonhomogeneous boundary conditions

$$
u^{(j)}( \pm 1)=\alpha_{ \pm \prime}^{j}, \quad j=0,1,2 .
$$

In such case we can proceed as.

Set

$$
V(x)=u(x)+\sum_{i=0}^{5} c_{i} x^{i}
$$

where $c_{i}, i=0,1, \ldots, 5$ are coefficients to be determined such that $V(x)$ satisfies the homogeneous boundary conditions, namely:

$$
V^{(j)}( \pm 1)=0, \quad j=0,1,2
$$

Therefore the set of coefficients $\left\{c_{i}, i=0, \ldots, 5\right\}$ are determined by solving the following system of six equations:

$$
\sum_{i=j}^{5}( \pm 1)^{i-j}(i-j+1){ }_{j} c_{i}=-\alpha_{ \pm \prime}^{j}, \quad j=0,1,2 .
$$

The system of (3.36) is equivalent to the following matrix equation:

$$
M c=-\alpha,
$$

where $\mathbf{c}=\left(c_{0}, c_{1}, \ldots, c_{5}\right)^{T}$ is the vector of unknowns, $\boldsymbol{\alpha}=\left(\alpha_{+}^{0}, \alpha_{-}^{0}, \ldots, \alpha_{+}^{5}, \alpha_{-}^{5}\right)^{T}$, and $M=$ $\left(m_{k j}\right)_{0 \leq k, j \leq 5}$ is a nonsingular matrix of order six and is given explicitly by

$$
\left(\begin{array}{cccccc}
1 & 1 & 1 & 1 & 1 & 1 \\
1 & -1 & 1 & -1 & 1 & -1 \\
0 & 1 & 2 & 3 & 4 & 5 \\
0 & 1 & -2 & 3 & -4 & 5 \\
0 & 0 & 2 & 6 & 12 & 20 \\
0 & 0 & 2 & -6 & 12 & -20
\end{array}\right)
$$

The transformation (3.34) turns the nonhomogeneous boundary conditions (3.36) into the homogeneous boundary conditions (3.35). Hence it suffices to solve the following modified one-dimensional sixth-order differential equation:

$$
-V^{(6)}(x)+\sum_{q=1}^{6} \delta_{q} \eta_{6-q} V^{(6-q)}(x)=f^{*}(x), \quad x \in(-1,1),
$$


subject to the homogeneous boundary conditions (3.35), and

$$
f^{*}(x)=f(x)+\sum_{q=1}^{6} \delta_{q} \eta_{6-q} \sum_{j=6-q}^{5} c_{j}(j+q-5)_{6-q} x^{j+q-6}
$$

Now, with the aid of the relation (see, [25])

$$
\begin{gathered}
x^{\ell}=\sum_{j=0}^{\ell} D_{\ell, j} R_{j}^{(3,3)}(x), \\
D_{\ell, j}=\frac{2^{j} \ell !(2 j+7)(j+1)_{3}}{n !} \sum_{i=0}^{\ell-j} \frac{(-2)^{i}(j+4)_{i}}{i !(\ell-i-j) !(j+7)_{i+1}(i+j+8)_{j}},
\end{gathered}
$$

we can write $f^{*}(x)$ as

$$
f^{*}(x)=f(x)+\sum_{q=1}^{6} \delta_{q} \eta_{6-q} \sum_{j=6-q}^{5} c_{j}(j+q-5)_{6-q} \sum_{k=0}^{j+q-6} D_{j+q-6, k} R_{k}^{(3,3)}(x) .
$$

If we apply Galerkin method to the modified equation (3.39), we get the following system of equations:

$$
\left(B+\sum_{q=1}^{6} \eta_{6-q} G_{6-q}\right) \mathbf{a}=\mathbf{f}^{*}
$$

where $\mathbf{f}^{*}=\left(f_{0}^{*}, f_{1}^{*}, \ldots, f_{N-6}^{*}\right)^{T} ; f_{k}^{*}=\left(f^{*}(x), R_{k}^{(3,3)}(x)\right)_{w} / h_{k}^{3,3}$, and the nonzero elements of the matrices $B$ and $G_{2 n-q}, 1 \leq q \leq 6$ are given explicitly as in Theorem 3.5.

\section{Two-Dimensional Sixth-Order Equations}

In this section, we consider the basis functions $\phi_{k}(x)$ as defined in (3.7) to solve numerically the two-dimensional even-order equations

$$
-\Delta^{3} u(x, y)+\sum_{r=0}^{2} \gamma_{r}(-\Delta)^{r} u(x, y)=f(x, y), \quad \text { in } \Omega,
$$

subject to the homogeneous boundary conditions

$$
\frac{\partial^{i}}{\partial x^{i}} u( \pm 1, y)=\frac{\partial^{i}}{\partial y^{i}} u(x, \pm 1)=0, \quad i=0,1,2,
$$


where $\Omega=(-1,1) \times(-1,1)$, the differential operator $\Delta$ is the well-known Laplacian defined by $\Delta \equiv \partial^{2} / \partial x^{2}+\partial^{2} / \partial y^{2}$, and $\gamma_{r}, 1 \leq r \leq n-1$ are constant, and $f(x, y)$ is a given function. $V_{N}^{2}$ such that

The symmetric generalized Jacobi-Galerkin approximation to (4.1)-(4.2) is to find $u_{N} \in$

$$
\left(-\Delta^{3} u_{N}, v\right)+\sum_{r=0}^{2} \gamma_{r}\left((-\Delta)^{r} u_{N}, v\right)=(f, v), \quad \forall v \in V_{N}^{2}
$$

It is clear that if we take $\phi_{k}(x)$ as defined in (3.7), then

$$
V_{N}^{2}=\operatorname{span}\left\{\phi_{i}(x) \phi_{j}(y), i, j=0,1, \ldots, N-6\right\} .
$$

Let us denote

$$
\begin{gathered}
u_{N}=\sum_{k=0}^{N-6} \sum_{j=0}^{N-6} u_{k j} \phi_{k}(x) \phi_{j}(y), \quad f_{k j}^{*}=\frac{1}{h_{k}^{3,3} h_{j}^{3,3}}\left(f(x, y), R_{k}^{(3,3)}(x) R_{j}^{(3,3)}(y)\right)_{w(x, y)^{\prime}} \\
U=\left(u_{k j}\right), \quad F^{*}=\left(f_{k j}^{*}\right), \quad k, j=0,1, \ldots, N-6, \\
w(x, y)=w^{(3,3)}(x) w^{(3,3)}(y)=\left(1-x^{2}\right)^{3}\left(1-y^{2}\right)^{3} .
\end{gathered}
$$

Taking $v(x, y)=\phi_{\ell}(x) \phi_{m}(y)$ in (4.3) for $\ell, m=0,1, \ldots, N-6$, then we find that (4.3) is equivalent to the following equation:

$$
\begin{aligned}
& \sum_{\ell, m=0}^{N-6}\left\{\left\{b_{i \ell} u_{\ell m} g_{j m}^{0}+3 g_{i \ell}^{4} u_{\ell m} g_{j m}^{2}+3 g_{i \ell}^{2} u_{\ell m} g_{j m}^{4}+g_{\ell m}^{0} u_{\ell m} b_{j m}\right\}\right. \\
& +\gamma_{2}\left\{g_{i \ell}^{4} u_{\ell m} g_{j m}^{0}+2 g_{i \ell}^{2} u_{\ell m} g_{j m}^{2}+g_{i \ell}^{0} u_{\ell m} g_{j m}^{4}\right\}+\gamma_{1}\left\{g_{i \ell}^{2} u_{\ell m} g_{j m}^{0}+g_{i \ell}^{0} u_{\ell m} g_{j m}^{2}\right\} \\
& \left.+\gamma_{0} g_{i \ell}^{0} u_{\ell m} g_{j m}^{0}\right\}=f_{i j}^{*}, \quad i, j=0,1 \ldots, N-6,
\end{aligned}
$$

which may be written in the matrix form:

$$
\begin{aligned}
& B U G_{0}^{T}+3 G_{4} U G_{2}^{T}+3 G_{2} U G_{4}^{T}+G_{0} U B^{T}+\gamma_{2}\left\{G_{4} U G_{0}^{T}+2 G_{2} U G_{2}^{T}+G_{0} U G_{4}^{T}\right\} \\
& +\gamma_{1}\left\{G_{2} U G_{0}^{T}+G_{0} U G_{2}^{T}\right\}+\gamma_{0} G_{0} U G_{0}^{T}=F^{*}
\end{aligned}
$$

where $U$ and $F^{*}$ are as defined in (4.6) and the nonzero elements of the matrices $B$ and $G_{6-q}, 1 \leq q \leq 6$, are those given as in Theorem 3.5. 
We can also rewrite (4.7) in the following form using the Kronecker matrix algebra (see, [26]):

$$
\begin{gathered}
L \mathbf{v} \equiv\left[B \otimes G_{0}+3 G_{4} \otimes G_{2}+3 G_{2} \otimes G_{4}+G_{0} \otimes B+\gamma_{2}\left\{G_{4} \otimes G_{0}+2 G_{2} \otimes G_{2}+G_{0} \otimes G_{4}\right\}\right. \\
\left.+\gamma_{1}\left\{G_{2} \otimes G_{0}+G_{0} \otimes G_{2}\right\}+\gamma_{0} G_{0} \otimes G_{0}\right] \mathbf{v}=\mathbf{f}^{*},
\end{gathered}
$$

where $\mathbf{f}$ and $\mathbf{v}$ are $F^{*}$ and $U$ written in a column vector, that is,

$$
\begin{aligned}
& \mathbf{f}^{*}=\left(f_{00}^{*}, f_{10}^{*}, \ldots, f_{N-6,0}^{*} ; f_{01}^{*}, f_{11}^{*}, \ldots, f_{N-6,1}^{*} ; \ldots ; f_{0, N-6}^{*}, \ldots, f_{N-6, N-6}^{*}\right)^{T}, \\
& \mathbf{v}=\left(u_{00}, u_{10}, \ldots, u_{N-6,0} ; u_{01}, u_{11}, \ldots, u_{N-6,1} ; \ldots ; u_{0, N-6}, \ldots, u_{N-6, N-6}\right)^{T}
\end{aligned}
$$

and $\otimes$ denotes the tensor product of matrices, that is, $A \otimes B=\left(A b_{i j}\right)_{i, j=0,1, \ldots, N-6}$.

A good review for the properties of the Kronecker product can be found in Graham [26] and Horn and Johnson [27].

In summary, the solution of (4.1)-(4.2) consists of the following six steps.

(i) Compute the matrices $F^{*}, B, G_{0}, G_{2}$, and $G_{4}$.

(ii) Compute the tensor products which appear in (4.9).

(iii) Write $F^{*}$ in a column vector $\mathbf{f}^{*}$.

(iv) Obtain a column vector $\mathbf{v}$ by solving (4.9).

(v) Rewrite a column vector $\mathbf{v}$ in the form $U$.

(vi) Find $u_{N}$.

Remark 4.1. Since $B$ is a diagonal matrix and each of the matrices $G_{6-q}, 1 \leq q \leq 6$, is sevenband at most, so the matrix $L$ in system (4.9) is $6(N-4)$-band at most, thus this system can be factorized by $L U$-decomposition and the number of operations necessary to construct this factorization is of order $6(N-5)(N-4)[12(N-4)+1]$, and the number of operations needed to solve the two triangular systems is of order $(N-5)[24(N-4)-3]$.

\section{Numerical Results}

We consider here three different examples.

Example 5.1. Consider the following one-dimensional sixth-order equation:

$$
\begin{gathered}
-u^{(6)}(x)+\sum_{q=1}^{6} \delta_{q} \eta_{6-q} u^{(6-q)}(x)=f(x), \quad x \in(-1,1), \\
u^{(j)}( \pm 1)=0, \quad j=0,1,2
\end{gathered}
$$


Table 2: Maximum pointwise error of $u-u_{N}$ for $N=8,12,16,20$.

\begin{tabular}{lcccccc}
\hline$N$ & $\eta_{i}$ & $E$ & $\eta_{i}$ & $E$ & $\eta_{i}$ & $E$ \\
\hline 8 & & $6.661 \cdot 10^{-4}$ & & $9.391 \cdot 10^{-4}$ & $8^{i+1}$ & $2.391 \cdot 10^{-3}$ \\
12 & 0 & $2.791 \cdot 10^{-8}$ & & $2.272 \cdot 10^{-18}$ & $12^{i+1}$ & $1.674 \cdot 10^{-7}$ \\
16 & 0 & $1.342 \cdot 10^{-13}$ & 1 & $1.269 \cdot 10^{-13}$ & $16^{i+1}$ & $1.241 \cdot 10^{-12}$ \\
20 & & $2.512 \cdot 10^{-16}$ & & $3.703 \cdot 10^{-16}$ & $20^{i+1}$ & $5.490 \cdot 10^{-16}$ \\
\hline
\end{tabular}

where $f(x)$ is chosen such that the exact solution of (5.1) is $u(x)=\left(1-x^{2}\right)^{3} \cos x$. The approximate spectral solution of (5.1) is given by

$$
u_{N}(x)=\sum_{k=0}^{N-6} a_{k} J_{k+6}^{(-3,-3)}(x)
$$

and the vector of unknowns $\mathbf{a}=\left(a_{0}, a_{1}, \ldots, a_{N-6}\right)^{T}$ is the solution of the system

$$
\left(B+\sum_{q=1}^{6} \eta_{6-q} G_{6-q}\right) \mathbf{a}=\mathbf{f}^{*},
$$

where the nonzero elements of the matrices $B$ and $G_{6-q}, 1 \leq q \leq 6$, are given explicitly as in Theorem 3.5 and $f_{k}^{*}=\left(f(x), R_{k}^{(3,3)}(x)\right)_{w} / h_{k}^{3,3}$.

Table 2 lists the maximum pointwise error $E$ for $u-u_{N}$ to (5.1), using SGJGM for various values $N$ and the set of coefficients $\left\{\eta_{i}, 0 \leq i \leq 5\right\}$.

Example 5.2. Consider the following BVP (see [28]):

$$
\begin{gathered}
y^{(6)}(x)+y(x)=6(2 x \cos x+5 \sin x), \quad x \in[-1,1], \\
y(-1)=y(1)=0, \\
y^{(1)}(-1)=y^{(1)}(1)=2 \sin (1), \\
y^{(2)}(-1)=-y^{(2)}(1)=-4 \cos (1)-2 \sin (1) .
\end{gathered}
$$

The exact solution of the above problem is

$$
y(x)=\left(x^{2}-1\right) \sin x
$$

Table 3 lists the maximum pointwise error $E=u-u_{N}$ using SGJGM for various valuesof $N$. This table shows that the best accuracy obtained by our method is $\left[2.256 \cdot 10^{-16}\right.$ for $\left.N=16\right]$ which is much better than the best accuracy obtained in Akram and Siddiqi [28] [3.81 $\left.10^{-16}\right]$. 
Table 3: Maximum pointwise error of $u-u_{N}$ for $N=8,10,12,14,16$.

\begin{tabular}{lc}
\hline$N$ & $E$ \\
\hline 8 & $8.301 \cdot 10^{-6}$ \\
10 & $2.247 \cdot 10^{-8}$ \\
12 & $4.499 \cdot 10^{-11}$ \\
14 & $7.707 \cdot 10^{-14}$ \\
16 & $2.256 \cdot 10^{-16}$ \\
\hline
\end{tabular}

Table 4: Maximum pointwise error of $u-u_{N}$ for $N=20,30,40$.

\begin{tabular}{ccccccccc}
\hline$N$ & $\gamma_{0}$ & $\gamma_{1}$ & $\gamma_{2}$ & SGJGM & $\gamma_{0}$ & $\gamma_{1}$ & $\gamma_{2}$ & SGJGM \\
\hline 20 & & & & $1.18 \cdot 10^{-3}$ & & & & $1.18 \cdot 10^{-3}$ \\
30 & 3 & 1 & 1 & $2.39 \cdot 10^{-9}$ & 0 & 0 & 0 & $1.18 \cdot 10^{-9}$ \\
40 & & & & $2.11 \cdot 10^{-14}$ & & & & $2.08 \cdot 10^{-14}$ \\
\hline
\end{tabular}

Example 5.3. Consider the two-dimensional sixth-order equation:

$$
-\Delta^{3} u+\gamma_{2} \Delta^{2} u-\gamma_{1} \Delta u+\gamma_{0} u=f(x, y), \quad \frac{\partial^{i} u}{\partial x^{i}}( \pm 1, y)=\frac{\partial^{i} u}{\partial y^{i}}(x, \pm 1)=0, \quad i=0,1,2,
$$

where $f(x, y)$ is chosen such that the exact solution of (5.6) is

$$
u(x, y)=\left(1-x^{2}\right)\left(1-y^{2}\right) \sin ^{2}(2 \pi x) \sin ^{2}(2 \pi y)
$$

In Table 4 , we list the maximum pointwise errors of $u-u_{N}$, using SGJGM with various choices of $N$.

\section{Concluding Remarks}

We have presented some efficient direct solvers for sixth-order equations in one- and twodimensions using the symmetric generalized Jacobi-Galerkin method. The algorithms are very efficient. In particular, we have found that, for some particular differential equations, the resulting systems of linear equations are diagonal. This, of course greatly simplify the numerical computations for these special cases. The use of symmetric generalized Jacobi polynomials leads to simplified analysis and very efficient numerical algorithms. Numerical results are presented which exhibit the high accuracy of the proposed algorithms.

\section{Acknowledgment}

The authors would like to thank the referee for his valuable comments and suggestions which improved the paper in its present form. 


\section{References}

[1] E. H. Doha, A. H. Bhrawy, and R. M. Hafez, "A Jacobi dual-Petrov-Galerkin method for solving some odd-order ordinary differential equations," Abstract and Applied Analysis, Article ID 947230, 21 pages, 2011.

[2] A. H. Bhrawy, A. S. Alofi, and S. I. El-Soubhy, "Spectral shifted Jacobi tau and collocation methods for solving fifth-order boundary value problems," Abstract and Applied Analysis, vol. 2011, Article ID 823273, 14 pages, 2011.

[3] G. Szegö, Orthogonal Polynomials, American Mathematical Society Colloquium Publication, 1985.

[4] G. E. Andrews, R. Askey, and R. Roy, Special Functions, vol. 71, Cambridge University Press, Cambridge, UK, 1999.

[5] J. P. Boyd, Chebyshev and Fourier Spectral Methods, Dover Publications, Mineola, NY, 2nd edition, 2001.

[6] D. Funaro, Polynomial approximation of differential equations, vol. 8 of Lecture Notes in Physics, Springer, Berlin, Germany, 1992.

[7] C. Canuto, M. Y. Hussaini, A. Quarteroni, and T. A. Zang, Spectral Methods in Fluid Dynamics, Springer, New York, NY, USA, 1989.

[8] A. Boutayeb and E. H. Twizell, "Numerical methods for the solution of special sixthorder boundaryvalue problems," International Journal of Computer Mathematics, vol. 45, no. 3, pp. 207-223, 1992.

[9] S. Chandrasekhar, Hydrodynamic and Hydromagnetic Stability, Clarendon Press, Oxford, UK, 1961.

[10] R. P. Agarwal, Boundary Value Problems for Higher Order Differential Equations, World Scientific, Singapore, 1986.

[11] A. H. Bhrawy, "Legendre-Galerkin method for sixth-order boundary value problems," Journal of the Egyptian Mathematical Society, vol. 17, no. 2, pp. 173-188, 2009.

[12] A. Lamnii, H. Mraoui, D. Sbibih, A. Tijini, and A. Zidna, "Spline collocation method for solving linear sixth-order boundary-value problems," International Journal of Computer Mathematics, vol. 85, no. 11, pp. 1673-1684, 2008.

[13] S. S. Siddiqi and E. H. Twizell, "Spline solutions of linear sixth-order boundary-value problems," International Journal of Computer Mathematics, vol. 60, no. 3-4, pp. 295-304, 1996.

[14] M. El-Gamel, J. R. Cannon, and A. I. Zayed, "Sinc-Galerkin method for solving linear sixth-order boundary-value problems," Mathematics of Computation, vol. 73, no. 247, pp. 1325-1343, 2004.

[15] B.-Y. Guo, J. Shen, and L.-L. Wang, “Optimal spectral-Galerkin methods using generalized Jacobi polynomials," Journal of Scientific Computing, vol. 27, no. 1-3, pp. 305-322, 2006.

[16] E. H. Doha and W. M. Abd-Elhameed, "Efficient spectral-Galerkin algorithms for direct solution of second-order equations using ultraspherical polynomials," SIAM Journal on Scientific Computing, vol. 24, no. 2, pp. 548-571, 2002.

[17] E. H. Doha and W. M. Abd-Elhameed, "Efficient spectral ultraspherical-dual-Petrov-Galerkin algorithms for the direct solution of $(2 n+1)$ th-order linear differential equations," Mathematics and Computers in Simulation, vol. 79, no. 11, pp. 3221-3242, 2009.

[18] E. H. Doha and A. H. Bhrawy, "Efficient spectral-Galerkin algorithms for direct solution of fourthorder differential equations using Jacobi polynomials," Applied Numerical Mathematics, vol. 58, no. 8, pp. 1224-1244, 2008.

[19] E. H. Doha, W. M. Abd-Elhameed, and A. H. Bhrawy, "Efficient spectral ultraspherical-Galerkin algorithms for the direct solution of 2nth-order linear differential equations," Applied Mathematical Modelling, vol. 33, no. 4, pp. 1982-1996, 2009.

[20] E. H. Doha, W. M. Abd-Elhameed, and Y. H. Youssri, "Efficient spectral-Petrov-Galerkin methods for third- and fifth-order differential equations using general parameters generalized Jacobi polynomials," Quaestiones Mathematicae, vol. 218, no. 15, pp. 7727-7740, 2012.

[21] M. Abramowitz and I. A. Stegun, Eds., Handbook of Mathematical Functions, vol. 55 of Applied Mathematical Series, National Bureau of Standards, New York, NY, USA, 1970.

[22] E. H. Doha, "The coefficients of differentiated expansions and derivatives of ultraspherical polynomials," Computers and Mathematics with Applications, vol. 21, no. 2-3, pp. 115-122, 1991.

[23] E. H. Doha, "On the coefficients of integrated expansions and integrals of ultraspherical polynomials and their applications for solving differential equations," Journal of Computational and Applied Mathematics, vol. 139, no. 2, pp. 275-298, 2002.

[24] M. Schatzman and J. Taylor, Numerical Analysis: A Mathematical Introduction, Clarendon Press, Oxford, UK, 2002.

[25] E. H. Doha, "On the construction of recurrence relations for the expansion and connection coefficients in series of Jacobi polynomials," Journal of Physics A, vol. 37, no. 3, pp. 657-675, 2004. 
[26] A. Graham, Kronecker Product and Matrix Calculus With Applications, Ellis Horwood, London, UK, 1981.

[27] R. A. Horn and C. R. Johnson, Topics in Matrix Analysis, Cambridge University Press, Cambridge, UK, 1991.

[28] G. Akram and S. S. Siddiqi, "Solution of sixth order boundary value problems using non-polynomial spline technique," Applied Mathematics and Computation, vol. 181, no. 1, pp. 708-720, 2006. 


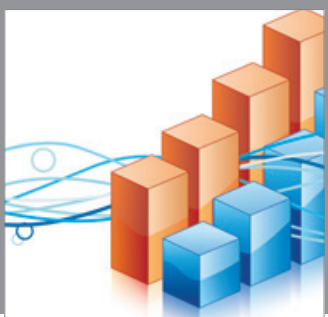

Advances in

Operations Research

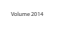

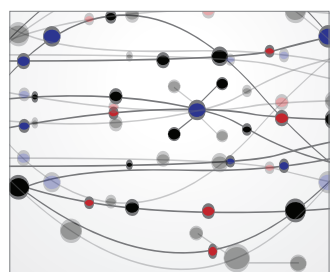

\section{The Scientific} World Journal
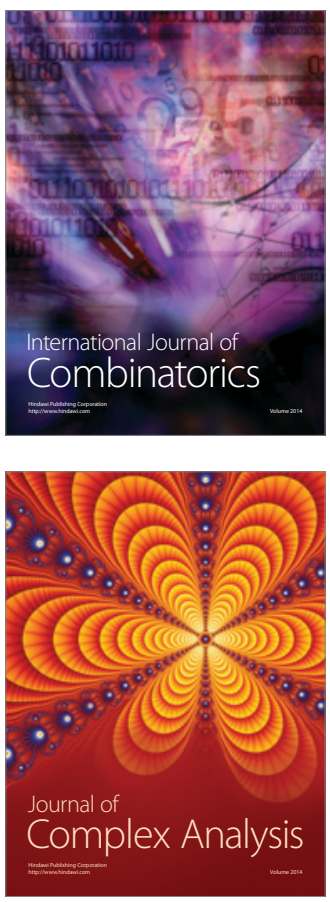

International Journal of

Mathematics and

Mathematical

Sciences
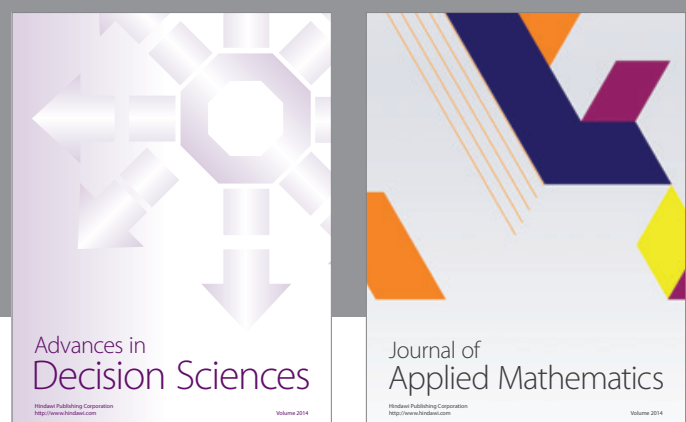

Journal of

Applied Mathematics
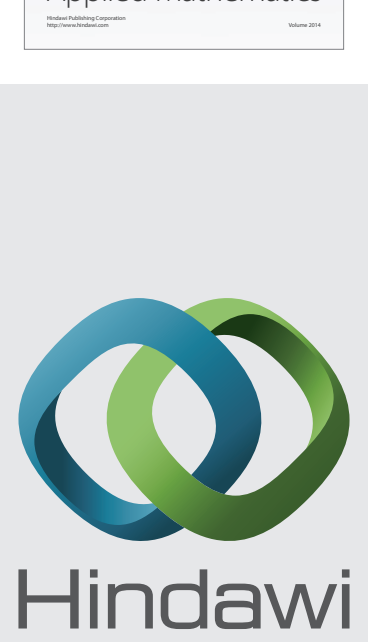

Submit your manuscripts at http://www.hindawi.com
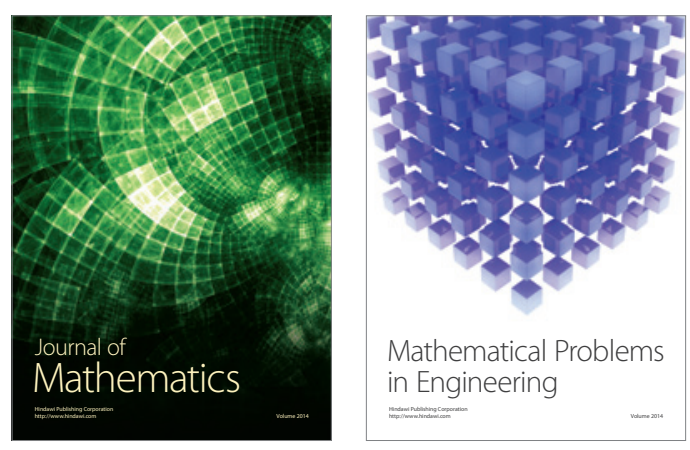

Mathematical Problems in Engineering
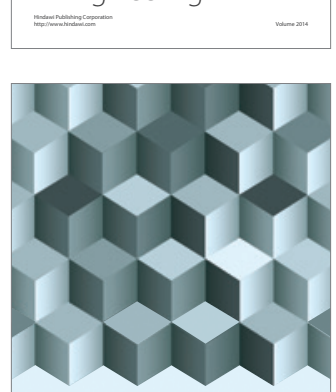

Journal of

Function Spaces
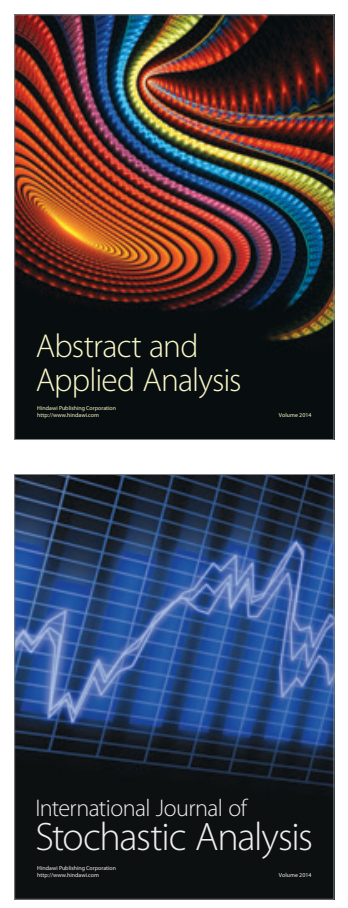

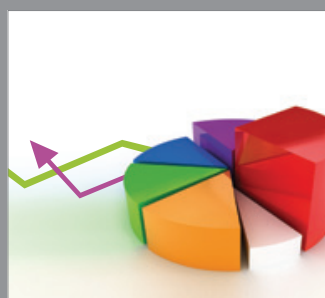

ournal of

Probability and Statistics

Promensencen
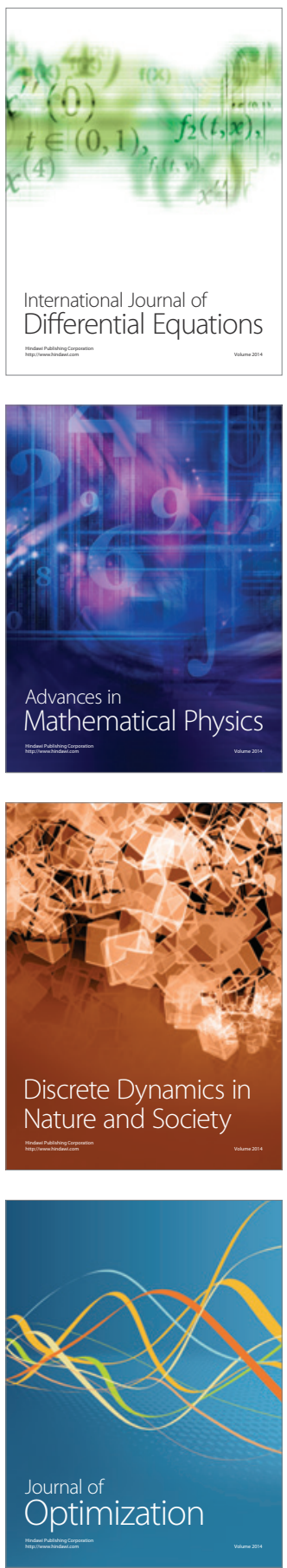